\title{
A comparison of GOCE and drifter-based estimates of the North Atlantic steady- state surface circulation
}

Article

Accepted Version

Bingham, R. J., Haines, K. and Lea, D. (2015) A comparison of GOCE and drifter-based estimates of the North Atlantic steady-state surface circulation. International Journal of Applied Earth Observation and Geoinformation, 35 (A). pp. 140-150. ISSN 0303-2434 doi:

https://doi.org/10.1016/j.jag.2014.03.012 Available at https://centaur.reading.ac.uk/36481/

It is advisable to refer to the publisher's version if you intend to cite from the work. See Guidance on citing.

Published version at: http://www.sciencedirect.com/science/article/pii/S0303243414000622

To link to this article DOI: http://dx.doi.org/10.1016/j.jag.2014.03.012

Publisher: Elsevier

All outputs in CentAUR are protected by Intellectual Property Rights law, including copyright law. Copyright and IPR is retained by the creators or other copyright holders. Terms and conditions for use of this material are defined in the End User Agreement. 


\section{CentAUR}

Central Archive at the University of Reading

Reading's research outputs online 


\title{
A comparison of GOCE and drifter-based estimates of the North Atlantic steady-state surface circulation
}

\author{
Rory J Bingham ${ }^{\mathrm{a}}$, Keith Haines ${ }^{\mathrm{b}}$, Daniel Lea ${ }^{\mathrm{c}}$ \\ ${ }^{a}$ School of Geographical Sciences, University of Bristol, Bristol, BS8 1SS, United \\ Kingdom \\ ${ }^{b}$ University of Reading, Reading, RG6 6AH, United Kingdom \\ ${ }^{c}$ Met Office, Exeter, EX1 3PB, United Kingdom
}

\begin{abstract}
Over the last decade, due to the Gravity Recovery And Climate Experiment (GRACE) mission and, more recently, the Gravity and steady state Ocean Circulation Explorer (GOCE) mission, our ability to measure the ocean's mean dynamic topography (MDT) from space has improved dramatically. Here we use GOCE to measure surface current speeds in the North Atlantic and compare our results with a range of independent estimates that use drifter data to improve small scales. We find that, with filtering, GOCE can recover $70 \%$ of the Gulf Steam strength relative to the best drifterbased estimates. In the subpolar gyre the boundary currents obtained from GOCE are close to the drifter-based estimates. Crucial to this result is careful filtering which is required to remove small-scale errors, or noise, in the computed surface. We show that our heuristic noise metric, used to determine the degree of filtering, compares well with the quadratic sum of mean sea surface and formal geoid errors obtained from the error variancecovariance matrix associated with the GOCE gravity model. At a resolution of $100 \mathrm{~km}$ the North Atlantic mean GOCE MDT error before filtering is 5
\end{abstract}


$\mathrm{cm}$ with almost all of this coming from the GOCE gravity model.

Keywords:

\section{Introduction}

The ocean's mean dynamic topography (MDT) is the surface expression of the horizontal pressure gradients associated with the ocean's steady-state circulation. From it, the geostrophic surface currents of the world's oceans can be diagnosed. These currents span a wide range of scales from global circulations, pivotal in the regulation of Earth's climate, to sub-mesoscale flows, knowledge of which is crucial for optimising the resource potential of the marine environment. Clearly then, accurate measurement of the ocean's MDT over its full range of spatial scales is an important scientific and practical goal.

While there exist a number of approaches to estimating the MDT, the focus in this paper is the geodetic method, whereby a global geoid $N$ is removed from an altimetric mean sea surface $H$ to leave only that part related to geostrophic ocean dynamics (Hughes and Bingham, 2008). This has the simple mathematical expression: $\eta=H-N$.

Accurate determination of the geoid is the limiting factor for the geodetic method. Early attempts to measure the MDT using satellite observations delivered poor results due to the low accuracy of early gravity field models (e.g. Tai and Wunsch, 1983; Denker and Rapp, 1990; Nerem et al., 1990). Over the last decade, however, the Gravity Recovery and Climate Experiment (GRACE; Tapley et al. (2004)) mission has produced a step change in our ability to measure Earth's global gravity field, and thus the global MDT, 
from space (e.g. Jayne et al., 2003; Tapley et al., 2003). However, GRACE has as its main focus the time variable gravity field and its design is such that it performs best at longer wavelengths, thus limiting the spatial scales of the MDT that may be resolved.

Launched in March 2009, the Gravity and steady-state Ocean Circulation Explorer (GOCE) satellite (Drinkwater et al., 2003), has been designed to measure the smaller spatial scales of Earth's gravity required to more fully resolve important features of the ocean's mean circulation, such as the narrow boundary currents. The pre-launch objective of the GOCE mission was an accuracy of $1 \mathrm{~cm}$ at spatial scales of $100 \mathrm{~km}$. Although, as we shall see below, this has yet to be achieved, a number of studies have found that GOCE has further improved our ability to measure the ocean's circulation from space (e.g. Bingham et al., 2011; Knudsen et al., 2011; Volkov and Zlotnicki, 2012).

Due to a number of remaining error sources, geodetic MDTs are usually filtered before ocean currents are calculated. A common approach is to use a Gaussian, or similar, spatial averaging filter (e.g. Jayne et al., 2003; Knudsen et al., 2011). While easy to implement, such isotropic filters in addition to removing noise also attenuate the MDT signal, particular in regions where there are steep gradients corresponding to strong currents; the features we are most interested in measuring. An alternative MDT filtering method based on non-linear diffusion has been shown by Bingham (2010) to better preserve these oceanographically important features when compared with conventional isotropic filtering.

Our aim here is to assess the ability of GOCE to measure the surface currents of the North Atlantic relative to a range of independent estimates 
which rely on in-situ drifter data. We do this for currents derived from both a filtered and an unfiltered GOCE MDT. In the case of the filtered MDT, establishing an objective criterion by which the degree of filtering required can be decided is crucial to realising the full potential from GOCE. We employ a method based on a heuristic metric of noise. We show that this metric is consistent with independently obtained error fields for the geoid and MSS. In doing so, we also determine their relative contributions to the MDT error budget. Even with careful filtering, however, we still find that if our concern is measuring the main current systems then a better approach may be to avoid filtering and chose a truncation that gives the best signal to noise ratio.

\section{The GOCE MDT and currents}

To compute the GOCE MDT (GMDT) and associated geostrophic surface current speeds for the North Atlantic we use the CLS11 mean sea surface (MSS; Schaeffer et al. (2012)). This MSS has been computed using 16 years of altimetry observations, including data from the TOPEX/POSEIDON, ERS2, GFO and JASON-1 missions, the ENVISAT mean profile and data from the two 168-day non-repeat cycles of the geodetic phase of ERS-1. The CLS11 MSS is estimated on a 2 arc-minute grid using a local inverse method, which also provides an estimation of the error field. Although the MSS is computed with 16 years of observations, the data is adjusted so that the MSS, and, therefore, the MDT computed from it, refers to the 1993-1999 time-mean. Note, variations of the geoid over time are considered negligible in comparison to those of the MSS and so the time-mean period of the MDT 
is overwhelmingly determined by the MSS.

From the CLS11 MSS we subtract a geoid determined from a GOCE gravity model. The GOCE High-level Processing Facility (HPF) is responsible for delivering the Earth gravity models to the user community (Koop et al., 2007). Within the HPF three processing strategies have been adopted, each with a distinct approach to generating the gravity model from the basic satellite-to-satellite tracking and gradiometer observations. An overview is provided by Pail et al. (2010). Here we use the third generation gravity model obtained by the so-called timewise approach (henceforth GTIM3) using one year of GOCE observations covering the period November 2009 to April 2011. Uniquely, the timewise approach is based purely on observations from GOCE. This model is freely available from http://icgem.gfzpotsdam.de/ICGEM/ICGEM.html.

The GMDT is calculated by the spectral method, as described in Bingham et al. (2008). The distinguishing feature of this method is that the MSS is first represented as a set of spherical harmonic coefficients. This allows the MDT to be computed up to a given harmonic degree $L \leq 250: \eta_{L}=H_{L}-N_{L}$. The upper limit of 250 is set by the GTIM3 gravity model which is defined to this degree and order. Note, however, that the GTIM3 gravity model is weakly regularised from degree 180 onwards (Pail et al., 2011). Effectively, this acts as a low-pass filter, not only suppressing noise in the high-degree coefficients but also attenuating the short-wavelength signal. This will, to some extent, limit the oceanographic signal that can be recovery for spatial scales less than $111 \mathrm{~km}$ and lead to some geoid omission error contamination 


\section{of the GMDT.}

By ameliorating the impact on the MDT of geoid omission error (that part of the gravity field not resolved by the model) and other numerical errors, such as Gibbs fringes, one advantage of the spectral method is that it permits a crucial conflict of errors to be explored. By increasing $L, \mathrm{MDT}$ omission error can be reduced; MDT gradients will be enhanced and finer scale features of the MDT may potentially be resolved. This is clearly seen for the GMDT as we move from $\eta_{30}$ (Figure 1a) to $\eta_{170}$ (Figure 1h). A conflict arises, however, because the impact of commission errors (particularly, as we shall see below, geoid commission errors) on the MDT grows with increasing $L$, and this tends to obscure any improvement due to the expected decrease in MDT omission error. This is particularly evident for the GMDT as we move from $\eta_{190}$ (Figure 1i) to $\eta_{250}$ (Figure 11).

\section{INSERT FIG 1 HERE}

Our primary interest in this study is ocean surface currents. Geostrophic surface current speeds are obtained through differentiation of $\eta_{L}$ :

$$
U_{L}=g\left|\nabla \eta_{L}\right| / f
$$

where $f$ is the Coriolis parameter, and $g$ is acceleration due to gravity. Being derived from the gradient of $\eta_{L}$, the spectral content of $U_{L}$ is shifted towards the higher degree terms. Hence, maximising $L$ becomes even more important for resolving ocean currents. Yet, as Fig. 2 makes clear, the differentiation also amplifies the noise in $\eta_{L}$, a problem increasingly exacerbated toward the equator due to the latitudinal dependence of $f$.

INSERT FIG 2 HERE 


\section{Errors and filtering}

\subsection{Informal errors and filtering}

Figure 2 suggests the need for filtering to remove noise before useful current speeds can be estimated from the GMDT. As discussed previously by Bingham et al. (2008), the severity of filtering should be carefully chosen to remove noise without unnecessarily attenuating the oceanographic signal. With the non-linear diffusive filtering approach used here (Bingham, 2010), the filtering severity is controlled by the number of iterations $I$ over which the filter is run. Bingham et al. (2011) find $I$ by minimising the root mean squared (RMS) difference between the currents derived from the filtered GMDT and an independent reference MDT. A metric such as this can, with a number of caveats, be thought of as a heuristic, or informal, MDT error estimate.

Adopting a similar approach, here we define an informal GMDT error for a given $L$ according to: $E_{L}^{\eta}=<\eta_{L}-\eta^{R}>$, where $\eta^{R}$ is some reference MDT, with $\langle *>$ representing the RMS difference of quantity $*$ computed in a $10^{\circ} \times 10^{\circ}$ window surrounding each grid point. To provide an independent reference we use an MDT estimate published by Niiler et al. (2003) (which we denote by NMM03). For our purposes it is useful that this MDT is not based on gravity data. Rather, it is derived primarily from in-situ drifter data corrected for non-geostrophic motions, including Ekman transport and inertial motion. It is defined on a global 0.5 degree grid and covers the period 1992-2003. To ensure consistence with the GMDT whose time mean period of 1993-99 is set by the period over which the MSS is computed, the NMM03 MDT is adjusted using AVISO sea level anomalies so that it refers to 
the same time-mean period. This is achieved simply by calculating the 19922003 mean of the 1993-99 referenced sea level anomalies and subtracting this mean from the NMM03 MDT.

It is important to note that the use of the NMM03 MDT does not imply that it is error free. If it were then there would of course be no need for the geodetic approach and missions such as GOCE. The important feature is that the error characteristics of the NMM03 MDT are quite different from those of the GMDT. For large spatial scales in particular it is expected that the GMDT will be more accurate. Indeed, the analysis presented below confirms this expectation. In contrast, as we move to shorter spatial scales the drifter-based MDT will have much smaller errors than the unfiltered GMDT. However, since the corrections applied to the drifter data will not be perfect and the drifter data coverage is not homogeneous - this is the one of the main arguments for the geodetic method - even the small scales in NMM03 will not be totally error free. For this reason the NMM03 MDT provides an upper bound on our estimate of the GMDT error.

Figure 3a (dashed red), shows the North Atlantic mean of $E_{L}^{\eta}$ with $\eta^{R}$ provided by the NMM03 MDT. Initially, $E_{L}^{\eta}$ falls with increasing $L$ due to a reduction in MDT omission error. $E_{L}^{\eta}$ reaches a minimum for $L \approx 110$, at which point the growth in MDT commission error begins to outweigh the reduction in MDT omission error. If, before computing $E_{L}^{\eta}$, we first express the reference MDT as a set of spectral coefficients and then project back into the spatial domain with expansion to degree $L\left(\eta^{R} \rightarrow \eta_{L}^{R}\right)$, differences due to MDT omission error can be largely negated (Figure 3a, solid red). In this case, $E_{L}^{\eta}$ approximates the upper bound on the MDT commission error, since 
it will also include errors in the NMM03 MDT, particularly at the largest spatial scales where we expect the geodetic approach to be superior.

\section{INSERT FIG 3 HERE}

Figure 3 b shows the equivalent differences for geostrophic current speeds: $E_{L}^{U}=<U_{L}-U_{L}^{R}>$, with reference current speeds, $U^{R}$ or $U_{L}^{R}$, computed from $\eta^{R}$ (dashed red) or from $\eta_{L}^{R}$ (solid red). Just as for $E_{L}^{\eta}$, with $U^{R}$ providing the reference, $E_{L}^{U}$ initially falls with increasing $L$, before rising at an increasing rate until an almost linear rate of growth is obtained. However, the initial drop in $E_{L}^{U}$ is much smaller than for $E_{L}^{\eta}$ and the minimum point occurs for lower $L$. This reflects the aforementioned shift in spectral content - both signal and error - of $U_{L}$ towards shorter spatial scales. Again, with $U_{L}^{R}$ as the reference the omission error component can be largely negated, and we take this as an upper bound on the commission component of the geostrophic current speed error.

Since our primary motivation here is to estimate ocean currents, for any given $L$, the minimisation of $E_{L}^{U}$ can be used to determine the number of iterations $I=I_{\min }$ over which the diffusive filter should be run. GMDTs $\eta_{L}$ were calculated for $0<L \leq L_{\max }=250$. The diffusive filter was then applied to each $\eta_{L}$ for 1000 iterations. The iteration minimising $E_{L}^{U}$ was then taken as $I_{\text {min }}$, with this providing the optimally filtered MDT $\bar{\eta}_{L}$ and associated geostrophic current speeds $\bar{U}_{L}$ for a given $L$.

Having obtained the optimally filtered fields, retrospective informal error estimates can be derived according to: $\bar{E}_{L}^{\eta}=<\eta_{L}-\bar{\eta}_{L}>$ and $\bar{E}_{L}^{U}=<$ $U_{L}-\bar{U}_{L}>$ (Figure 3, dashed blue). In this case the error estimates $\bar{E}_{L}^{*}$ are not contaminated by errors in the NMM03 based references, but reflect the 
combined effect of the noise, removed by the filter, and any error introduced by the filter's attenuation of the signal. However, these estimates cannot account for MDT errors that are not removed by the filter. Thus, $\bar{E}_{L}^{\eta}$ and $\bar{E}_{L}^{U}$ represent lower bounds on the MDT and geostrophic current speed commission error, plus the deleterious impact of the filter on the MDT. Correcting for this filter-induced error, as described immediately below, in the GMDT, and in a similar fashion for the currents, gives the true lower bounds for the $\eta_{L}$ and $U_{L}$ commission error (Figure 3, solid blue).

The negative consequence of filtering is the attenuation of the MDT gradients and loss of finer scale detail which filtering causes. The diffusive filter is specifically designed to reduce this. However, it does not eliminate it. Clearly for a given number of filter iterations the attenuation of an MDT will depend on its initial spectral content or resolution - how much signal there is to lose - which depends on $L$. Hence, the NMM03 MDT was used to model this filter-induced error according to: $E_{L}^{f}=<\eta_{L}^{R}-\bar{\eta}_{L}^{R}>$, where $\bar{\eta}_{L}^{R}$ is the spectrally truncated NMM03 MDT diffusively filtered over the same number of iterations as $\bar{\eta}_{L}$. $E_{L}^{f}$ will not be a perfect estimate of the filter induced error in $\bar{\eta}_{L}$ because it will also include the error in $\bar{\eta}_{L}^{R}$ removed by the filter. However, this error is only a small part of what is already a small correction to the initial lower bound error estimate.

The convergence of $E_{L}^{*}$ and $\bar{E}_{L}^{*}$ in Figure 3 confirms that these informal error spectra are indeed dominated by the GMDT noise for $100<L$. Another way of putting this is that as we move towards shorter spatial scales the error in the NMM03 MDT is far outweighed by the noise in the GMDT; What the filter removes from the GMDT is almost identical to the difference 
between the NMM03 MDT and the unfiltered GMDT. Moving in the opposite direction towards larger spatial scales, however, the two error metrics diverge, with the metric based on the NMM03 MDT approximately $2 \mathrm{~cm}$ larger than the lower bound at $L=50$, corresponding to spatial scales of $400 \mathrm{~km}$. This is in line with our expectation that, without employing gravity data, the errors in the NMM03 MDT will be greater than in the GMDT at the largest spatial scales. The analysis below confirms this to be the case.

Because the differences between the lower and upper error bounds are due to long wavelength differences between the GOCE and NMM03 MDTs, the difference between the two error bounds almost vanishes when current speeds are considered. Figure 3b demonstrates that magnitude of the residual between the currents obtained from the unfiltered GMDT and the NMM03 MDT is almost identical to the noise in the GMDT current field suppressed by the filter. In other words, for a given truncation the difference between the NMM03 and GMDT currents is much smaller than the amplitude of the noise in the GMDT currents. Note also that the change due to the correction for the filter-induced error is extremely small, which shows that the attenuation of the currents by the filter is small in comparison to the noise removed by the filter.

Using the error metrics $E_{L}^{\eta}=<\bar{\eta}_{L}-\eta^{R}>$ and $E_{L}^{U}=<\bar{U}_{L}-U^{R}>$ (Figure 3 , green), we find the point at which the reduction in MDT and current speed omission error, resulting from increasing $L$, is outweighed by the deleterious impact of the filter, which grows as the noise increases. In fact, this only just starts to become a problem after $L=220$, and so we take $\bar{\eta}_{220}$ and $\bar{U}_{220}$ as our best possible estimates of the MDT and geostrophic currents with the 
data and filtering method used (see Figure 4).

INSERT FIG 4 HERE

\subsection{Assessment of the informal GMDT error}

To provide as assessment of the informal error metrics derived above, we now consider to what extent the informal GMDT errors can be accounted for in terms of the errors in the constituent fields. Assuming these errors to be independent, the MDT error budget can be written thus:

$$
\sigma_{\eta}=\sqrt{\sigma_{N}^{2}+\sigma_{H}^{2}}
$$

where $\sigma_{N}$ is the geoid error and $\sigma_{H}$ is the MSS error.

The formal geoid errors for the GTIM3 gravity model were evaluated from the variance-covariance matrix at truncations $L=10$ to 250 in 10 degree intervals as described in Appendix A. Figure 5 (cyan) shows that the area mean formal geoid errors over the North Atlantic increase with truncation from a few millimetres at $L=10$ to $15 \mathrm{~cm}$ at $L=250$.

While the CLS11 MSS is supplied with a formal error field, for our analysis we require an estimate of MSS error as a function of $L$. This was obtained in a manner analogous to the method used to obtain the informal MDT error estimates: $E_{L}^{H}=<H_{L}-H_{L}^{R}>$, where $H^{R}$ is a reference MSS. Here we use the DTU10 MSS, which is computed from a combination of 17 years (19932009) of satellite altimetry from a total of 8 satellites (Andersen and Knudsen, 2009). The North Atlantic mean of $E_{L}^{H}$ is shown in Figure 5 (magenta). At $L=250$ its value is $1.7 \mathrm{~cm}$, close to the North Atlantic mean formal error of $1.9 \mathrm{~cm}$ (magenta square).

INSERT FIG 5 HERE 
Figure 5b compares the sum of the geoid and MSS errors (green) with the upper (red) and lower (blue) informal estimates of GMDT error derived above. The lower informal estimate and the error sum are in close agreement. This serves as confirmation of both the GTIM3 formal error and the retrospective informal GMDT error estimate. The difference between the two informal GMDT error estimates is an measure of the reference MDT (NMM03) error, which, as expected, grows with increasing spatial scale.

\section{Assessment of GOCE currents}

\subsection{Comparison after filtering}

Having obtained an optimum estimate of the MDT and associated surface currents using the GOCE data and filtering method, we now compare the results with a range of independent MDTs which use a combination of satellite and in-situ data. In addition to the NMM03 MDT we compare the GMDT against two further products: (i) The CLS09 MDT refers to the period 1993-99. It uses in-situ drifter and hydrographic data together with altimetric sea level anomaly data and information from an MDT derived from an ocean model to refine an initial geodetic MDT estimate based on a MSS and a GRACE geoid (Rio et al., 2011). (ii) The Maximenko et al. (2009) MDT (MAX11 for brevity) is also a synthesis of a large-scale geodetic MDT, derived from a GRACE geoid and a MSS, and small-scale information provided by drifter, NCEP wind and altimetry data. MAX11 covers the period 1992-2002 and the version used here is dated January 7, 2011. For consistency, the MAX11 MDT is also adjusted to the 1993-99 period in the same way as NMM03. 


\section{INSERT FIG 6 HERE}

The geostrophic surface current fields obtained from the drifter-based MDTs (DMDTs) are shown in Figure 6 (top row) and look similar to each other and to the GMDT. Figure 6 (bottom row) shows the differences between GMDT and DMDTs. With the exception of the lower reaches of the Gulf Stream, the GMDT generally gives stronger current speeds than MAX11. Overall, the CLS09 and NMM03 DMDTs give stronger currents and resolve more detail than the GMDT. Exceptions to this occur for the sub-polar boundary currents which appear weaker than in the GMDT, especially for CLS09.

\section{INSERT TABLES 1,2,3 HERE}

This first impression is confirmed by the values given in Table 1 where we see that for the Florida Current, which marks the initiation of the Gulf Stream (position 1), the GMDT estimate of $42 \mathrm{~cm} \mathrm{~s}^{-1}$ is much less than the 66-72 $\mathrm{cm} \mathrm{s}^{-1}$ speeds given the DMDTs. For position 2, along the boundary following path of the Gulf Stream, the GMDT speed of $51 \mathrm{~cm} \mathrm{~s}^{-1}$ is less than the $62-95 \mathrm{~cm} \mathrm{~s}^{-1}$ range given by the DMDTs, while for position 3 , the GMDT speed of $61 \mathrm{~cm} \mathrm{~s}^{-1}$ exceeds the $47 \mathrm{~cm} \mathrm{~s}^{-1}$ given by MAX11, but is 15-27 $\mathrm{cm} \mathrm{s}^{-1}$ lower than the other two DMDTs.

East of $55^{\circ} \mathrm{W}$, the strong zonal flow of the Gulf Stream breaks down and makes its way north and then east through a series of smaller scale structures including the Mann Eddy, which mark the beginning of the NAC. This detail is most clearly resolved in the CLS09 and NMM03 products. In comparison, while some of the finer-scale structure can be discerned in the GMDT currents, it is much weaker than in either of these DMDTs. The same 
is true of the MAX11 MDT where, despite the inclusion of drifter data, the detail is also attenuated relative to the other DMDTs. CLS09 and NMM03 give the current speed at the northern edge of the Mann Eddy (position 4) as 53 and $50 \mathrm{~cm} \mathrm{~s}^{-1}$ respectively. The speed given the GMDT is $6-9 \mathrm{~cm} \mathrm{~s}^{-1}$ less than this, but greater than the $31 \mathrm{~cm} \mathrm{~s}^{-1}$ given by MAX11. Where the NAC flows eastward (position 5) we find that the GMDT gives a similar current speed $\left(9 \mathrm{~cm} \mathrm{~s}^{-1}\right)$ to that of the DMDTs $\left(7-10 \mathrm{~cm} \mathrm{~s}^{-1}\right)$.

To set these point values in context, Figure 7a shows the current speeds along the entire northward-flowing warm path (plotted in Figure 4d as curve intersecting circles 1-5). Downstream of location 2 the current speeds given by the GMDT follow quite closely those of the CLS09 and NMM03 products, with the MAX11 currents being much weaker. The attenuation of the finer-scale structure of the NAC relative to CLS09 and NMM03 can be seen downstream of location 4 .

Considering the section from position 1 to position 3 , we find that the GMDT has an average speed of $60 \mathrm{~cm} \mathrm{~s}^{-1}, 68-70 \%$ of the speed given by CLS09 and NMM03, but 110\% of the MAX11 speed (see Table 2). The average speed for the GMDT along the sections defined by positions 3 and 4 is $38 \mathrm{~cm} \mathrm{~s}^{-1}$, which is $92-99 \%$ of the speeds given by the CLS09 and NMM03 MDTS, while for the section defined by positions 4 and 5 it is $14 \mathrm{~cm} \mathrm{~s}^{-1}$, which is $65-69 \%$ of the strength given by CLS09 and NMM03.

\section{INSERT FIG 7 HERE}

Considering next the predominately southward flowing cold route (plotted in Figure 4d as curve intersecting circles 6-9) we find current speeds which overall most closely resemble those given by the MAX11 MDT (see Figure 
7b). Next most similar are the CLS09 currents, with those from NMM03 being much noisier. Considering some key locations along this path, for the East Greenland Current (position 6), the GMDT gives a current speed of 25 $\mathrm{cm} \mathrm{s}^{-1}$, close to MAX11 $\left(26 \mathrm{~cm} \mathrm{~s}^{-1}\right)$ and CLS09 $\left(22 \mathrm{~cm} \mathrm{~s}^{-1}\right)$, but less than the $38 \mathrm{~cm} \mathrm{~s}^{-1}$ given by NMM03. For the West Greenland Current (position 7) the GMDT current speed of $12 \mathrm{~cm} \mathrm{~s}^{-1}$ is a little greater than the $9 \mathrm{~cm} \mathrm{~s}^{-1}$ speeds given by MAX11 and CLS09, but less than the $21 \mathrm{~cm} \mathrm{~s}^{-1}$ given by NMM03. For the Labrador current (position 8) the GMDT estimate of 28 $\mathrm{cm} \mathrm{s}^{-1}$ is lower than the $38-45 \mathrm{~cm} \mathrm{~s}^{-1}$ range given by the DMDTs. Finally at the southern tip of the Labrador current (position 9), while the $21 \mathrm{~cm} \mathrm{~s}^{-1}$ current speed given by the GMDT is greater than the speeds given by CLS09 $\left(12 \mathrm{~cm} \mathrm{~s}^{-1}\right)$ and MAX11 $\left(20 \mathrm{~cm} \mathrm{~s}^{-1}\right)$, it is again less than the $31 \mathrm{~cm} \mathrm{~s}^{-1}$ given by NMM03. Considered over the entire sub-polar path, the GMDT has an average speed of $16 \mathrm{~cm} \mathrm{~s}^{-1}$. This is somewhat greater than the average speeds for CLS09 and MAX11, but only 87\% of the speed given by NMM03.

\subsection{Comparison without filtering}

The optimum GMDT estimate considered thus far is determined by finding the degree and order $L$ and number of filter iterations $I_{\text {min }}$ that minimise the RMS difference with the reference MDT over the entire basin. The large area is used to reduce the sensitivity of the filter to a particular choice of reference MDT. However, because the noise is relatively uniformly distributed across the basin, its reduction through filtering more strongly influences the RMS difference than the attenuation of currents which occupy a much smaller area of the domain. Thus the final, filtered solution may not be optimum if our concern is just the regions where the currents are strongest and where 
the signal to noise ratio is much better. Therefore, finally we consider the current speeds along the warm and cold paths that can be obtained from the GOCE data without filtering.

Taking the region of complex structure of the NAC as our reference, visual inspection of Figure 2 suggests a threshold value of $L \approx 170$ beyond which the amplitude of the noise starts to exceed the amplitude of the signal. A more quantitative assessment whereby we compute the RMS difference between the DMDTs and the unfiltered GMDTs for $L=0, \ldots, 250$ along the warm and cold paths confirms $L=170$ as approximately the optimum unfiltered truncation. Figure 8 also shows that the roughness of the GMDT current speed curve is comparable to that of the DMDTs. In fact, for the subpolar path it is still less.

The most dramatic change is found for position 1 where the current speed of $83 \mathrm{~cm} \mathrm{~s}^{-1}$ is now greater than the range given by the DMDTs. This shows that the attenuation due to filtering is most severe at the initiation of the Gulf Stream between positions 1 and 2. Following the sub-tropical path we see that only upstream of location 2 is the unfiltered $L=170$ GMDT substantial less than CLS09 or NMM03. Downstream of this location the GMDT is very close to CLS09 and NMM03. Without filtering, the GMDT is now 20 and 31 $\mathrm{cm} \mathrm{s}^{-1}$ less than CLS09 and NMM03 estimates at position 2 and $7 \mathrm{~cm} \mathrm{~s}^{-1}$ less than the CLS09 at position 3. The GMDT has an average speed of 75 $\mathrm{cm} \mathrm{s}^{-1}$ along the Gulf Stream path defined by positions 1 and 3, 86-88\% of the speeds given by CLS09 and NMM03, but substantially greater than the MAX11 speed (see Table 3). On the northern flank of the Mann Eddy (position 4) the GMDT estimate of $54 \mathrm{~cm} \mathrm{~s}^{-1}$ is close to the $50 \mathrm{~cm} \mathrm{~s}^{-1}$ and 
$53 \mathrm{~cm} \mathrm{~s}^{-1}$ estimates of NMM03 and CLS09. Along the section, defined by positions 3 and 4 the GMDT average speed of $42 \mathrm{~cm} \mathrm{~s}^{-1}$ is slightly greater than the speeds given by CLS09 and NMM03 and much greater than the speed given by MAX11. The speed of the eastward flowing NAC (position 5 ) is now $14 \mathrm{~cm} \mathrm{~s}^{-1}$, somewhat greater than the $7-10 \mathrm{~cm} \mathrm{~s}^{-1}$ range given by the DMDTs, and the average speed along the section defined by positions 4 and 5 is $20 \mathrm{~cm} \mathrm{~s}^{-1}$, the same as that given by CLS09.

Along the East Greenland Current (position 6) the GMDT gives a current speed of $32 \mathrm{~cm} \mathrm{~s}^{-1}$, greater than CLS09 $\left(22 \mathrm{~cm} \mathrm{~s}^{-1}\right)$ and MAX11 $\left(26 \mathrm{~cm} \mathrm{~s}^{-1}\right)$ and only a little less than NMM03 $\left(38 \mathrm{~cm} \mathrm{~s}^{-1}\right)$. Similar relative magnitudes are found at position 7. At position 8 on the Labrador Current the GMDT speed $\left(42 \mathrm{~cm} \mathrm{~s}^{-1}\right)$ lies within the $38-45 \mathrm{~cm} \mathrm{~s}^{-1}$ range given by the DMDTs, while at position 9 the GMDT speed $\left(25 \mathrm{~cm} \mathrm{~s}^{-1}\right)$ is again greater than CLS09 $\left(12 \mathrm{~cm} \mathrm{~s}^{-1}\right)$ and MAX11 $\left(20 \mathrm{~cm} \mathrm{~s}^{-1}\right)$, but less than NMM03 $\left(31 \mathrm{~cm} \mathrm{~s}^{-1}\right)$. The average sub-polar current speed of $23 \mathrm{~cm} \mathrm{~s}^{-1}$ given by the GMDT is, however, substantially greater than the average speeds given by all of the DMDTs. The NMM03 MDT stands out as being particularly noisy along this path suggesting errors due to the lack of in-situ data.

INSERT FIG 8 HERE

\section{Concluding discussion}

It is possible to use in-situ drifter data to determine a high resolution MDT without the need for gravity data (Niiler et al., 2003). However, such an approach is likely not reliable at large spatial scales. Therefore, in-situ drifter data is commonly used to improve the finer-scale resolution of an 
initial geodetic MDT estimate which can be considered more accurate at large spatial-scales (e.g. Rio et al., 2011; Maximenko et al., 2009). However, the space/time sampling of the ocean by drifters is sparse, drifters are prone to failure (Grodsky et al., 2011), and assumptions and corrections are required to isolate the geostrophic component of the surface current speeds measured by drifters. Therefore, drifters are far from an ideal way to estimate the time-mean circulation of the ocean. Thus, with respect to the ocean, the purpose of the GOCE mission can be thought of as being to relive as far as is possible the reliance on drifters to provide the finer-scale structure of the mean circulation.

The limiting factor in this endeavor is the growth in MDT noise as we push the geodetic MDT towards higher resolution. According to the informal estimates derived in this study the unfiltered GOCE MDT error for $L=$ $200 \approx 100 \mathrm{~km}$ when averaged over the North Atlantic basin is about $5 \mathrm{~cm}$, which translates to a $15 \mathrm{~cm} \mathrm{~s}^{-1}$ current speed error. Comparison with the formal geoid error obtained from the error covariance matrix supplied with the GOCE geoid and an informal estimate of the CLS11 MSS error suggests that almost all of the GMDT error is contributed by the geoid. This is somewhat higher than the hoped for nominal mission accuracy of $1 \mathrm{~cm}$ at scales of $100 \mathrm{~km}$.

Although, almost all of the noise can be removed by filtering, this is at the expense of attenuating the MDT gradients associated with the strong current systems and finer scale structures. With the diffusive filtering method used here this attenuation is much less than with conventional isotropic spatial average filtering. However, it is still significant. 
To quantify the attenuation we compared the current speeds obtained from the filtered GMDT with those from drifter-based MDTs. This comparison can also be thought of as an assessment of the additional signal that is contributed by the drifter data. In spite of the attenuation due to filtering, the current speeds from the GOCE MDT are almost everywhere superior to (stronger than) those obtained from the Maximenko et al. (2009) MDT, showing the care that must be taken in combining the geodetic and in-situ data if any benefit is to be gained from the latter. Given this relative lack of power in the MAX11 MDT, we consider CLS09 and NMM03 as more appropriate benchmarks against which the GMDT should be assessed.

The greatest attenuation of the filtered GMDT occurs for the Florida Current, with the current speed nearly half that obtained from the drifterbased products. However, this constricted and tightly curving current is unusual. Along the main path of the Gulf Stream the filtered GMDT currents are around $70 \%$ the strength of the currents given by NMM03 and CLS09. In other words, assuming the full signal is present in the unfiltered GMDT (a point we shall come back to), filtering attenuates the signal by about $30 \%$ along the path of the Gulf Stream.

At higher latitudes, the benefits derived from using drifter data are not as obvious. In fact, the boundary currents of the subpolar gyre are weaker in CLS09 and MAX11 than they are in the filtered GMDT, while those obtained from the NMM03 MDT appear incoherent along the path of the subpolar currents. These differences between the GMDT and drifter-based MDTs may be explained by the relative paucity of drifter data at higher latitudes and demonstrate the importance of the denser, more uniform sampling provided 
by geodetic approach.

While the filtered GMDT solution may provide the best foundation for a synthesis with drifter data, if our aim is just to obtain the best estimate of the current speeds in the major current systems, then, given the better signal to noise ratio where currents are strong, it may be more appropriate to determine current speeds without filtering the GMDT. We find that with no filtering, truncating the GMDT at $L=170$, corresponding to a resolution of $118 \mathrm{~km}$, gives the closest match to the drifter-based current speeds along the paths of the major current systems of the North Atlantic. If we go beyond this, any increase in signal is outweighed by the increase in noise.

Comparing the unfiltered $L=170$ GMDT with CLS09 and NMM03, we find that $86 \%$ of the Gulf Stream strength can be recovered from the geodetic approach using the GOCE data. Without filtering, the current strength of subpolar boundary currents are much stronger than those given by the CLS09 and MAX11 MDTS and are comparable or larger than those given by the NMM03 MDT, while still remaining much smoother. The error analysis allows us to assign an error of $9 \mathrm{~cm} \mathrm{~s}^{-1}$ to the unfiltered currents at $L=170$.

In summary, our analysis shows that using GOCE we can come close to capturing the full resolution of the ocean's steady-state circulation. The limiting factor is still filtering which attenuates the gradients associated with strong currents and finer-scale features. If drifter data is to be combined with a geodetic GOCE MDT then its contribution will be primarily to restore the signal lost by filtering. As the attenuation is related to the degree of filtering required, which, in turn, is dependent on geoid commission error, this error component should diminish as more GOCE data are collected and the geoid 
error is reduced. But even with just one year of observations in many regions GOCE improves upon what can be achieved with GRACE and drifter data.

\section{Appendix A. Error propagation}

The full error variance-covariance matrices for each gravity model are provided to the user community by the HPF (Pail et al. (2011); obtained from http://eo-virtual-archive1.esa.int/). Ultimately we are concerned to see how these formal errors are expressed in the MDT. To achieve this we must express the gravity field errors in terms of geoid error. To do this we use the error propagation tools developed by Balmino (2009). (The error propagation tools described in Balmino (2009) are obtained from https://earth.esa.int/web/guest/softwaretools.)

The theoretical description of the error propagation performed by the covhsmp routine to obtain the geoid error field is as follows: Let the gridded geoid be given by

$$
N(\lambda, \theta)=\mathbf{Y}^{T} X
$$

where $\lambda$ is longitude and $\theta$ is geocentric latitude and

$$
\mathbf{X}=\left\{C_{l m} ; S_{l m}\right\}
$$

are the spherical harmonic coefficients of degree $l$ and order $m$ of the earth gravity model, and

$$
\mathbf{Y}=\left\{f_{l m} P_{l m}(\sin \theta) \cos m \lambda ; f_{l m} P_{l m}(\sin \theta) \sin m \lambda\right\}
$$

are the usual spherical harmonic functions with

$$
f_{l m}=\frac{G M}{r \gamma}\left(\frac{R}{r}\right)^{l}
$$


where $G M$ is Earth's gravitational mass constant, $R$ is Earth's mean radius, $\gamma$ is normal gravity at the computation point and $r$ is radial distance. Then the corresponding error variance field is given by:

$$
\sigma_{N}^{2}(\lambda, \theta)=\mathbf{Y}^{T} \mathbf{\Gamma} \mathbf{Y}
$$

where $\boldsymbol{\Gamma}$ is the variance-covariance matrix of the GTIM3 gravity model with ordering consistent with $\mathbf{Y}$.

\section{References}

Andersen, O., Knudsen, P., 2009. The DNSC08 mean sea surface and mean dynamic topography. J. Geophys. Res. 114. doi:10.1029/2008JC005179.

Balmino, G., 2009. Efficient propagation of error covariance matrices of gravitational models: application to GRACE and GOCE. J. Geod. 83, 989-995.

Bingham, R., Haines, K., Hughes, C., 2008. Calculating the ocean's mean dynamic topography from a mean sea surface and a geoid. J. Atmos. Ocean. Tech. 25, 1808-1822. doi:10.1175/2008JTECHO568.1.

Bingham, R.J., 2010. Nonlinear anisotropic diffusive filtering applied to the ocean's mean dynamic topography. Rem. Sens. Lett. 1, 205-212. doi:10.1080/01431161003743165.

Bingham, R.J., Knudsen, P., Andersen, O., Pail, R., 2011. An initial estimate of the North Atlantic steady-state geostrophic circulation from GOCE. Geophys. Res. Lett. 38. doi:10.1029/2010GL045633. 
Denker, H., Rapp, R.H., 1990. Geodetic and oceanographic results from the analysis of 1 year of Geosat data. J. Geophys. Res. 95, 13,151-13,168. doi:10.1029/JC095iC08p13151.

Drinkwater, M., Floberghagen, R., Haagmans, R., Muzi, D., Popescu, A., 2003. GOCE: ESA's first Earth Explorer core mission. Space Science Reviews 108, 419-432.

Grodsky, S.A., Lumpkin, R., Carton, J., 2011. Spurious trends in global surface drifter currents. Geophys. Res. Lett. 38. URL: http://dx.doi.org/10.1029/2011GL047393, doi:10.1029/2011GL047393.

Hughes, C.W., Bingham, R.J., 2008. An oceanographer's Guide to GOCE and the Geoid. Ocean Sci. 4, 15-29.

Jayne, S., Wahr, J., Bryan, F., 2003. Observing ocean heat content using satellite gravity and altimetry. J. Geophys. Res. 108. doi:10.1029/2002JC001619.

Knudsen, P., Bingham, R., Andersen, O., Rio, M.H., 2011. A global mean dynamic topography and ocean circulation estimation using a preliminary GOCE gravity model. J. Geod. 85, 861-879.

Koop, R., Gruber, T., Rummel, R., 2007. The status of the GOCE highlevel processing facility (HPF), in: Proceedings of the 3rd GOCE User Workshop, ESRIN, Frascati.

Maximenko, N., Niiler, P., Rio, M.H., Melnichenko, O., Centurioni, L., Chambers, D., Zlotnicki, V., Galperin, B., 2009. Mean dynamic 
topography of the ocean derived from satellite and drifting buoy data using three different techniques. J. Atmos. Ocean. Tech. 26. doi:10.1175/2009JTECHO672.1.

Nerem, R.S., Tapley, B.D., Shum, C.K., 1990. Determination of the ocean circulation using Geosat altimetry. J. Geophys. Res. 95, 3163-3179. doi:10.1029/JC095iC03p03163.

Niiler, P., Maximenko, N., McWilliams, J., 2003. Dynamically balanced absolute sea level of the global ocean derived from near-surface velocity observations. Geophys. Res. Lett. 30. doi:10.1029/2003GL018628.

Pail, R., Bruinsma, S., Migliaccio, F., Forste, C., Goiginger, H., Schuh, W.D., Hock, E., Reguzzoni, M., Brockmann, J., Abrikosov, O., Veicherts, M., Fecher, T., Mayrhofer, R., Krasbutter, I., Sano, F., Tscherning, C., 2011. First GOCE gravity field models derived by three different approaches. J. Geod. 85, 819-843. doi:10.1007/s00190-011-0467-x.

Pail, R., Goiginger, H., Mayrhofer, R., Schuh, W.D., Brockmann, J., Krasbutter, I., Hock, E., Fecher, T., 2010. Goce gravity field model derived from orbit and gradiometry data applying the time-wise method., in: Proceedings of the ESA Living Planet Symposium, Bergen, Norway, European Space Agency.

Rio, M.H., Guinehut, S., Larnicol, G., 2011. New CNES-CLS09 global mean dynamic topography computed from the combination of grace data, altimetry, and in situ measurements. J. Geophys. Res. 116. doi:10.1029/2010JC006505. 
Schaeffer, P., Faugre, Y., Legeais, J.F., Ollivier, A., Guinle, T., Picot, N., 2012. The CNES-CLS11 global mean sea surface computed from 16 years of satellite altimeter data. Marine Geodesy 35, 3-19. doi:10.1080/01490419.2012.718231.

Tai, C.K., Wunsch, C., 1983. Absolute measurement by satellite altimetry of dynamic topography of the Pacific Ocean. Nature 301, 408-410. doi:10.1038/301408a0.

Tapley, B., Bettadpur, S., Watkins, M., Reigber, C., 2004. The gravity recovery and climate experiment: Mission overview and early gravity results. Geophys. Res. Lett. 31. doi:10.1029/2004GL019920.

Tapley, B., Chambers, D., Bettadpur, S., Ries, J., 2003. Large scale ocean circulation from the GRACE GGM01 geoid. Geophys. Res. Lett. 30. doi:10.1029/2003GL018622.

Volkov, D.L., Zlotnicki, V., 2012. Performance of GOCE and GRACEderived mean dynamic topographies in resolving Antarctic Circumpolar Current fronts. Ocean Dynamics 62, 893-905. doi:10.1007/s10236-0120541-9. 
Table 1: Current speeds $\left(\mathrm{cm} \mathrm{s}^{-1}\right)$ at nine locations marked in Figure 4.

\begin{tabular}{lccccccccc}
\hline \hline MDT & 1 & 2 & 3 & 4 & 5 & 6 & 7 & 8 & 9 \\
\hline GMDT (d/o=220; no filter) & 147 & 79 & 60 & 68 & 6 & 64 & 20 & 62 & 44 \\
GMDT (d/o=220; filtered) & 42 & 51 & 61 & 44 & 9 & 25 & 12 & 28 & 21 \\
GMDT (d/o=170; no filter) & 83 & 64 & 81 & 54 & 14 & 32 & 15 & 42 & 25 \\
CLS09 & 72 & 84 & 88 & 53 & 9 & 22 & 9 & 38 & 12 \\
MAX11 & 66 & 62 & 47 & 31 & 7 & 26 & 9 & 45 & 20 \\
NMM03 & 68 & 95 & 76 & 50 & 10 & 38 & 21 & 41 & 31 \\
\hline
\end{tabular}

Table 2: Average d/o=220 filtered GMDT current speeds along sections with end-points marked in Figure 4. GMDT current speeds in $\mathrm{cm} \mathrm{s}^{-1}$ and expressed in percent of drifterbased MDT current speeds.

\begin{tabular}{lcccc}
\hline \hline MDT & $1-3$ & $3-4$ & $4-5$ & $6-9$ \\
\hline GMDT $\left(\mathrm{cm} \mathrm{s}^{-1}\right)$ & 60 & 38 & 14 & 16 \\
CLS09 $(\%)$ & 70 & 92 & 69 & 104 \\
MAX11 (\%) & 110 & 140 & 98 & 108 \\
NMM03 (\%) & 68 & 99 & 65 & 87 \\
\hline
\end{tabular}

Table 3: Average $\mathrm{d} / \mathrm{o}=170$ unfiltered GMDT current speeds along sections with endpoints marked in Figure 4. GMDT current speeds in $\mathrm{cm} \mathrm{s}^{-1}$ and expressed in percent of drifter-based MDT current speeds.

\begin{tabular}{lcccc}
\hline \hline MDT & $1-3$ & $3-4$ & $4-5$ & $6-9$ \\
\hline GMDT $\left(\mathrm{cm} \mathrm{s}^{-1}\right)$ & 75 & 42 & 20 & 23 \\
CLS09 (\%) & 88 & 101 & 100 & 153 \\
MAX11 (\%) & 138 & 154 & 144 & 159 \\
NMM03 (\%) & 86 & 109 & 94 & 128 \\
\hline
\end{tabular}



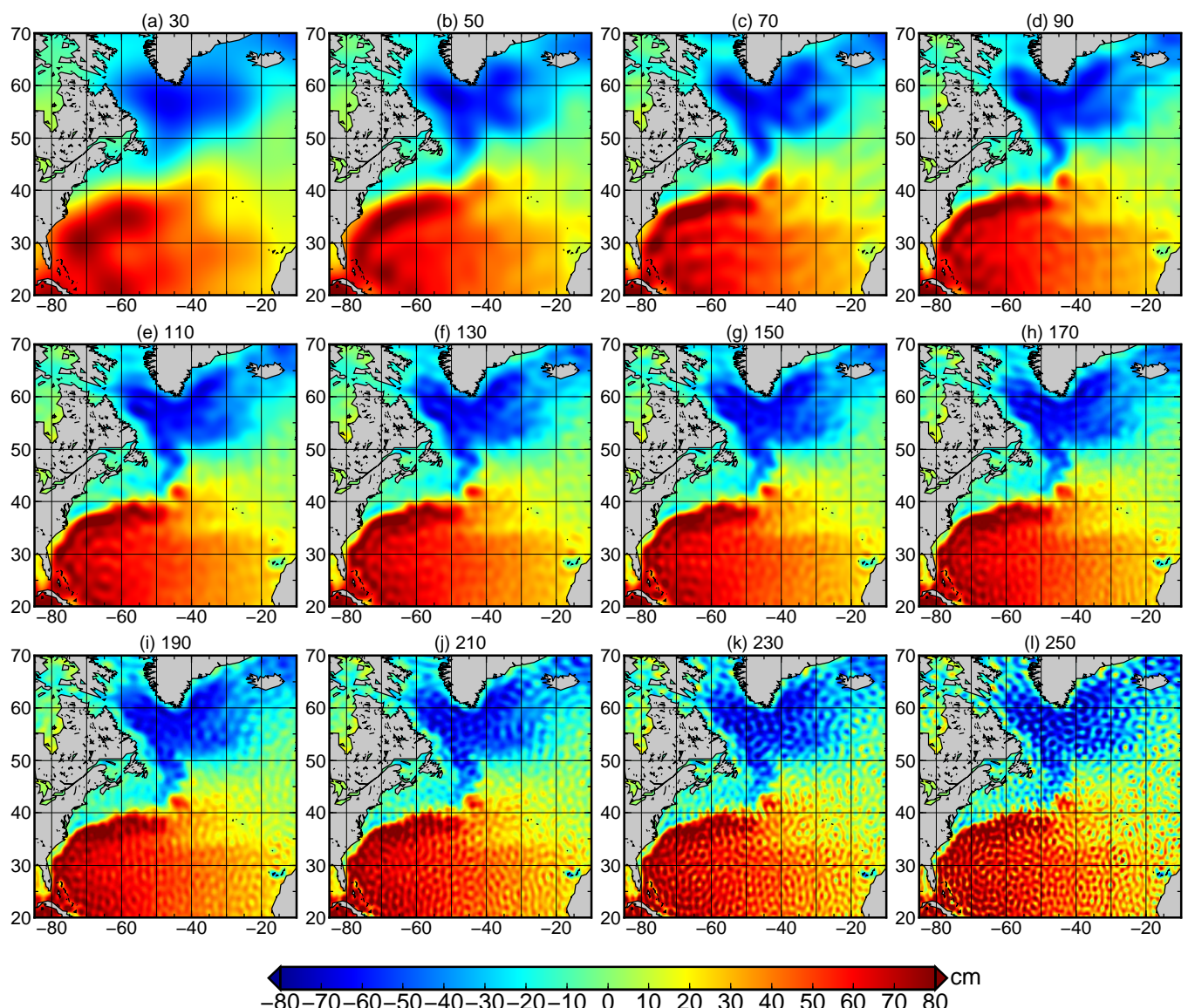

Figure 1: The mean dynamic topography (MDT) of the North Atlantic, based on the CLS01 mean sea surface and the 3rd generation GOCE time-wise gravity model, with truncations of the spherical harmonic expansion of the MDT ranging from $L=30$ to 250 in intervals of 20 . 

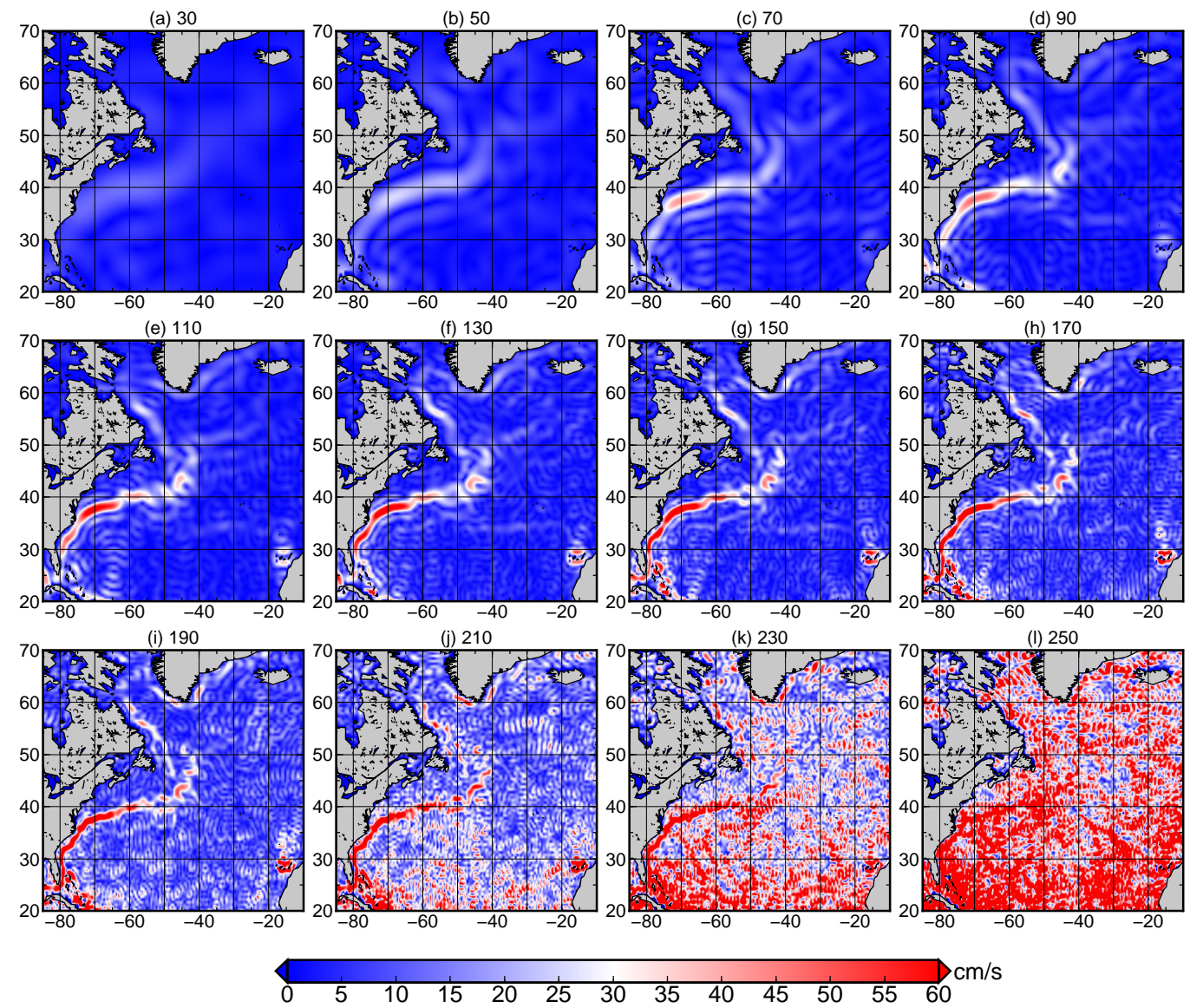

Figure 2: The mean geostrophic surface current speeds of the North Atlantic, obtained from the MDTs shown in Fig. 1 
(a)

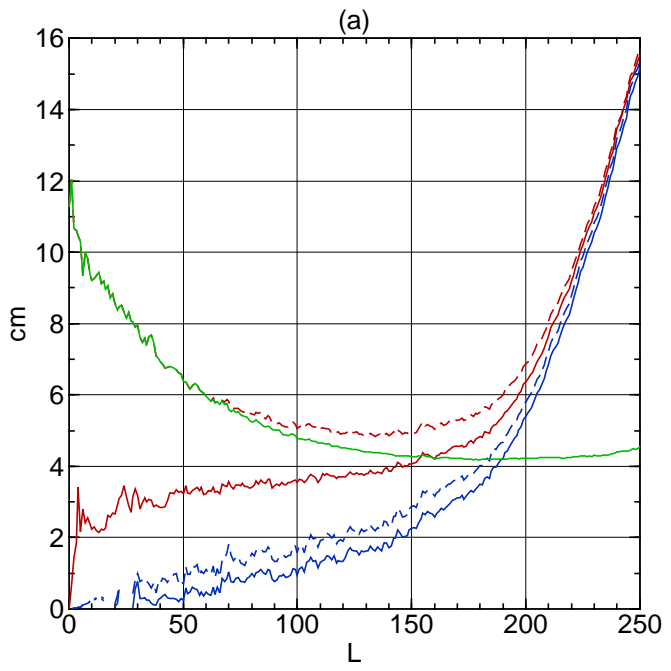

(b)

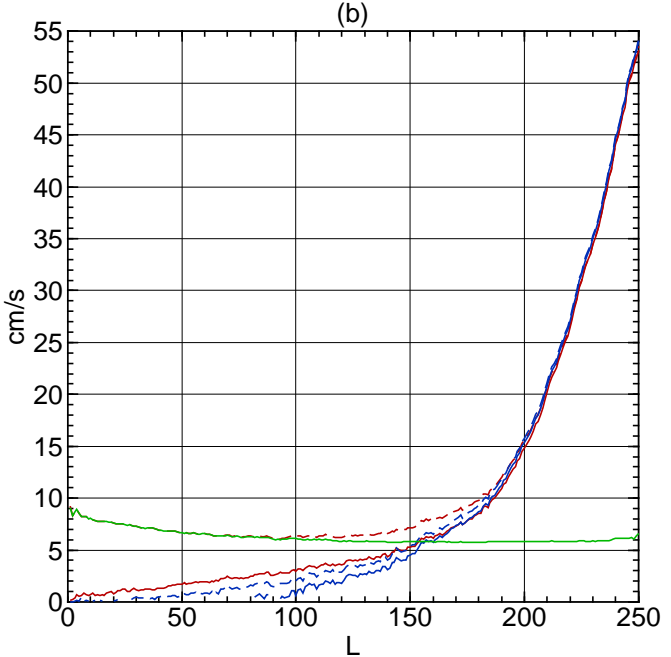

Figure 3: (a) Informal mean dynamic topography (MDT) error estimates (as described in text): Full MDT error (red dashed), upper bound on MDT commission error (red), lower bound on MDT commission error plus filter induced error (blue dashed), lower bound on MDT commission error (blue), and the full error for the optimally filtered MDT (green).(b) Repeating (a) for geostrophic current speeds. 

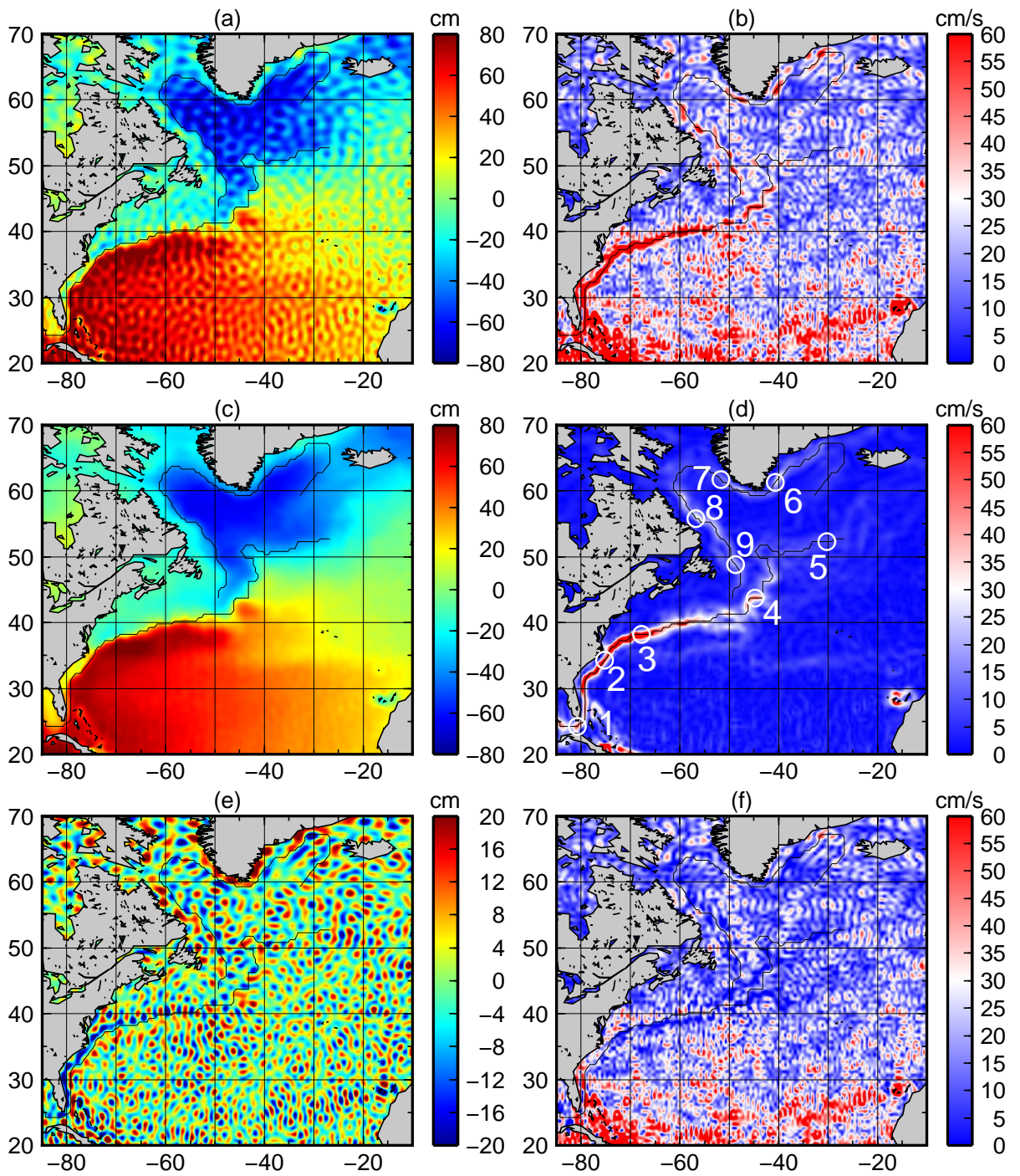

Figure 4: (a,b) The GOCE MDT for $L=220$, and associated geostrophic surface current speed field. (c,d) The optimally filtered GOCE MDT for $L=220$, and associated current field. (e,f) The difference between the filtered and unfiltered fields, i.e. what the filter removes. 

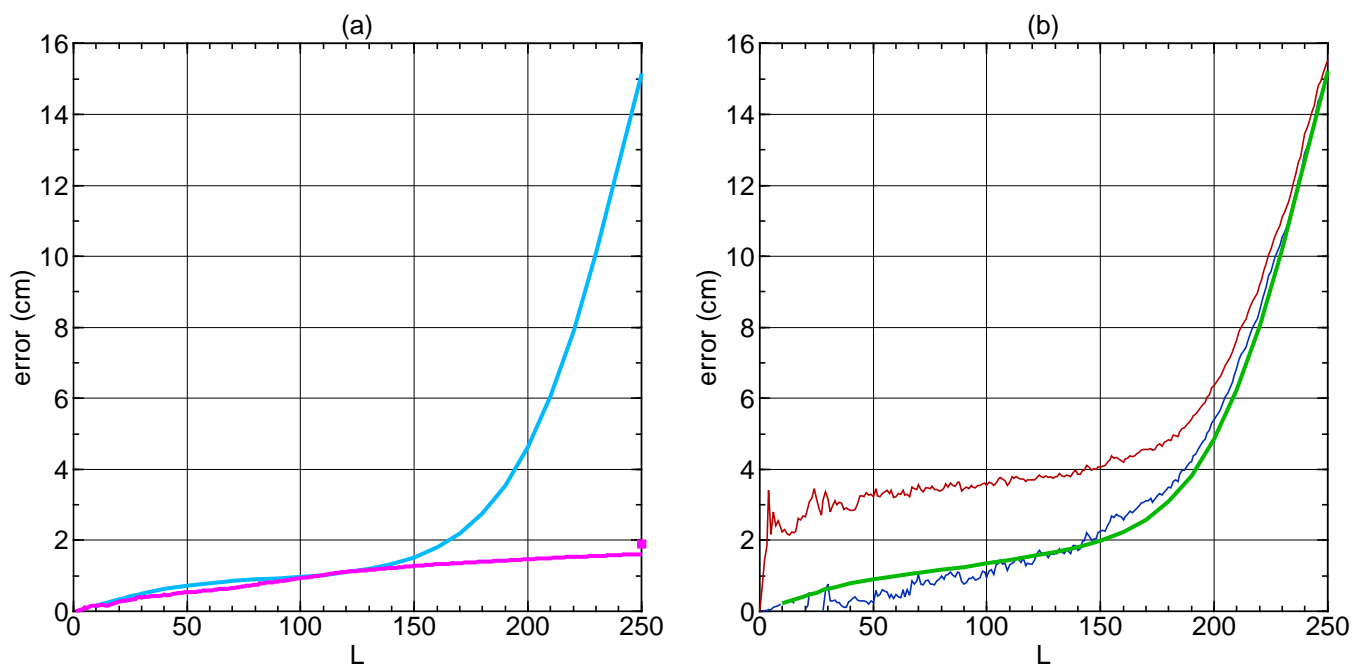

Figure 5: (a) North Atlantic mean formal errors for the geoid obtained from the third GOCE timewise gravity model (cyan). An informal estimate of the CLS11 MSS error based on the RMS difference between it and the DTU10 MSS (magenta). The area mean over the North Atlantic of the supplied CLS11 formal error field is marked by the solid square. (b) Upper (red) and lower (blue) bounds on the GMDT commission error (repeated from Figure 3a). The quadratic sum of the geoid and MSS errors (green). 
(a) CLS09

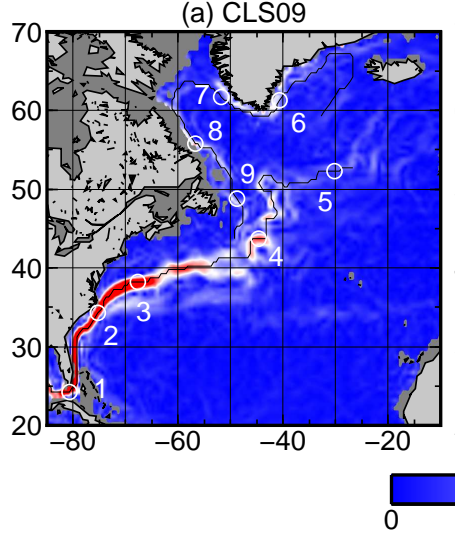

(d) CLS09-GMDT

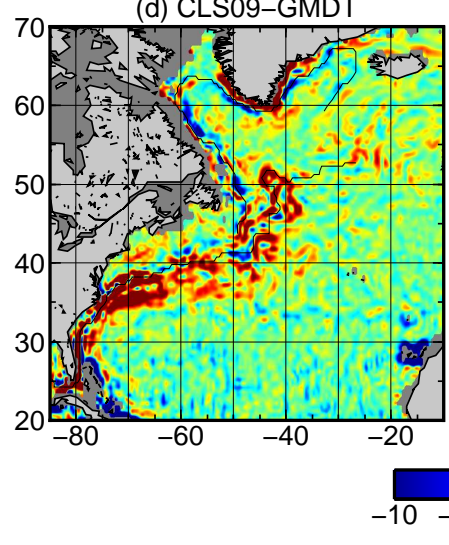

(b) MAX11
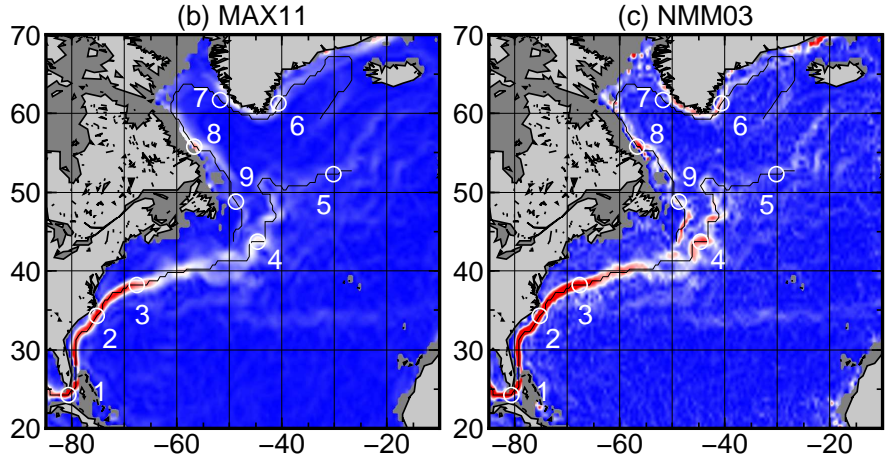

$\mathrm{cm} / \mathrm{s}$

\section{(e) MAX11-GMDT}

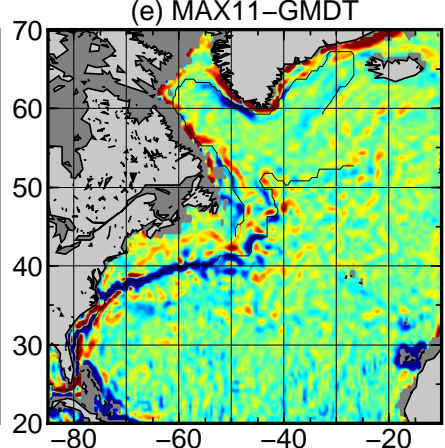

(f) NMM03-GMDT

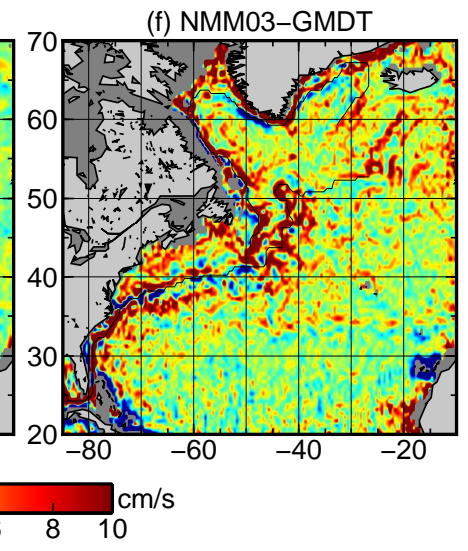

Figure 6: North Atlantic geostrophic surface current speeds obtained from the (a) CLS09 MDT, (b) the MAX11 MDT and (c) The NMM03 MDT. (d-f) The residual upon subtracting the GOCE MDT from the drifter-based MDTs. 

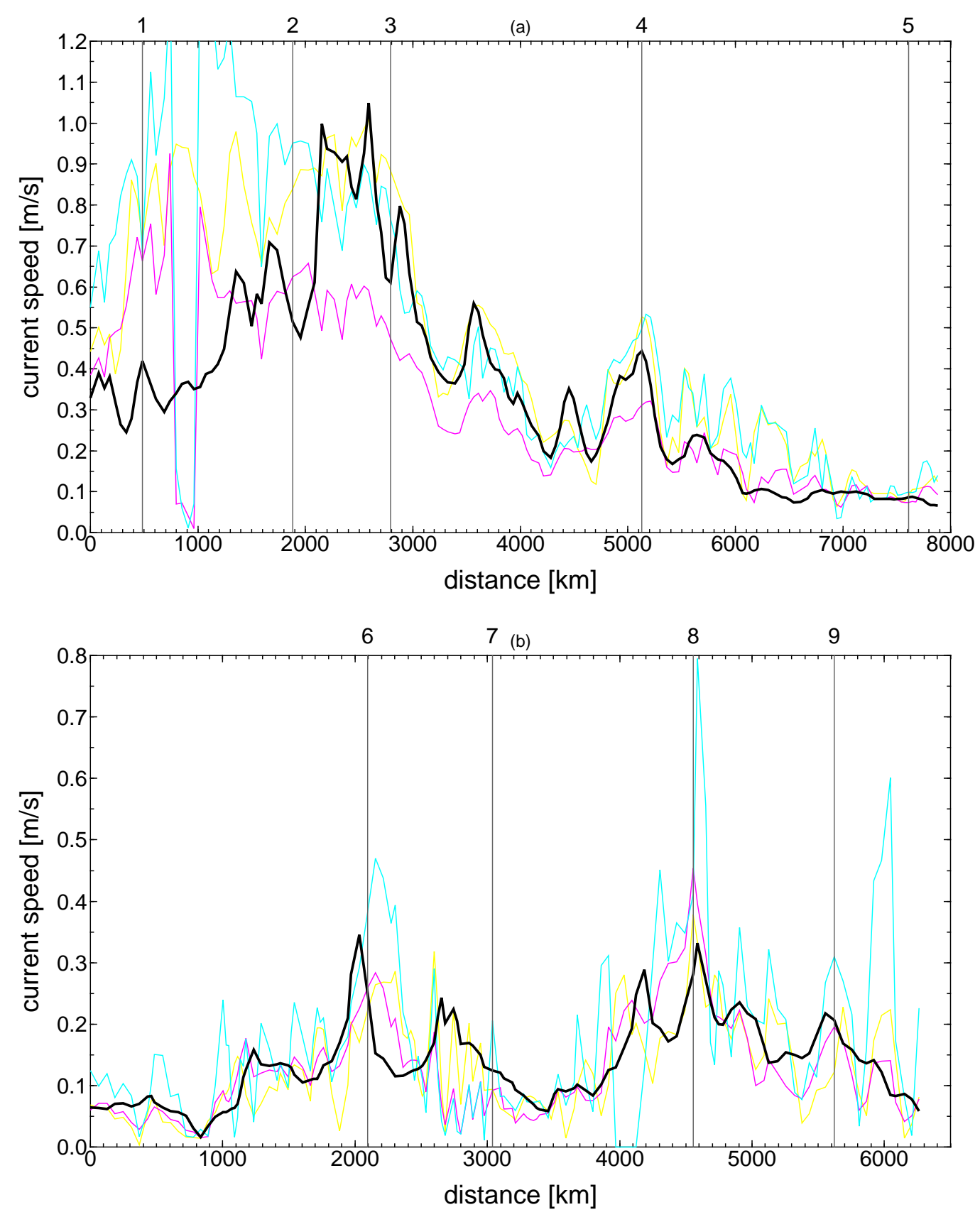

Figure 7: (a) Current speeds along the warm path marked in Figure 4d with current speeds given by the GOCE MDT shown in Figure 4d (black), the CLS09 MDT (yellow), the MAX11 MDT (magenta) and the NMM@3 MDT (cyan). (b) Repeating (a) but for the cold sub-polar path marked in Figure 4d. The vertical lines correspond to the positions marked on Figure 4d. 

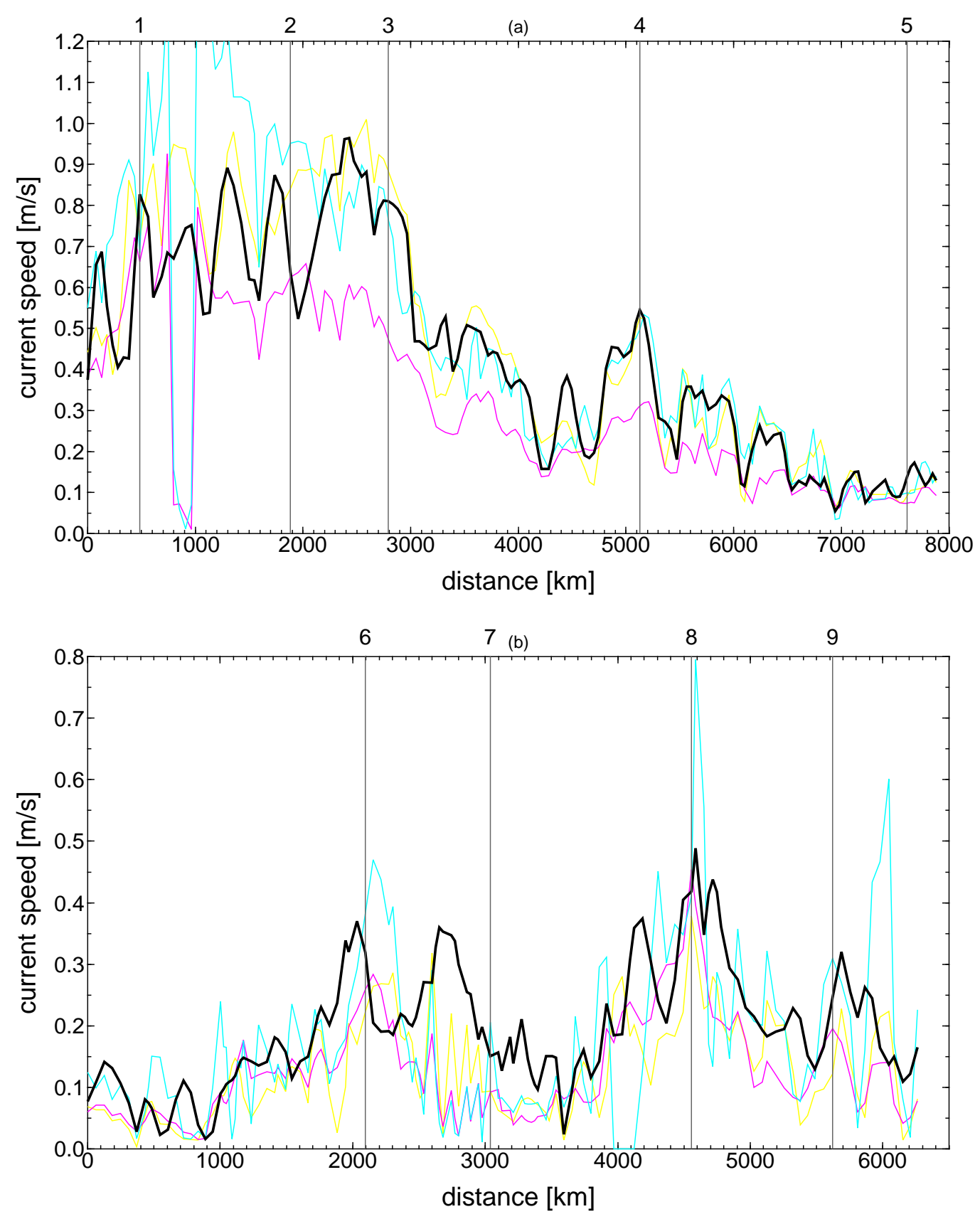

Figure 8: (a) Current speeds along the warm path marked in Figure $4 \mathrm{~d}$ with current speeds given by the unfiltered $L=170$ GOCE MDT shown in Figure 2h (black), the CLS09 MDT (yellow), the MAX11 MDT (magenta) and the NMM03 MDT (cyan). (b) Repeating (a) but for the cold sub-polar path marked in Figure 4d. The vertical lines correspond to the positions marked on Figure 4d. 